\title{
Utility of p63 and AMACR in Differentiating Benign and Malignant Prostatic Lesions
}

\author{
Ashily Koshy and Rupali Bavikar* \\ Department of Pathology, Dr. D. Y. Patil Medical College, Hospital and Research Centre, Dr. D. Y. Patil Vidyapeeth, Pune
}

\begin{abstract}
Background: Prostate cancer is the sixth most common cancer in the world. Since treatment options and prognosis of prostatic adenocarcinomas and benign lesions differ significantly, so, the current study using p63and AMACR was carried out with aim to evaluate the utility of immunohistochemistry in resolving ambiguous lesions of prostate.

Methods: The study was conducted on 130 prostatic specimens which included prostate biopsy, TURP and prostatectomy specimens. Routine hematoxylin and eosin staining and immunohistochemical staining using AMACR, p63 monoclonal antibody markerwere performed.

Results: Total of 130 cases of prostate samples were studied out of which benign lesions were seen in 102 cases (78.5\%) and malignant carcinoma in 28 cases $(21.5 \%)$. This study showedp63 had a sensitivity of $92.86 \%$ and specificity of $100 \%$ whereas AMACR has a sensitivity of $96.4 \%$ and specificity of $95 \%$. BPH with prostatitis was a common finding in majority of benign lesions. All cases of LGPIN 15 cases $(11.5 \%)$ were histologically associated with BPH, showing complete positivity in 12 cases (80\%) and partial positivity in 3 cases $(20 \%)$ with p63 immunostaining. HGPIN $(1.5 \%)$ were associated with prostatic adenocarcinoma showing focal positivity whereas adenocarcinoma showed complete negativity of p63 expression (100\%) and positive cytoplasmic staining with AMACR. Comparative study done between DRE, PSA, final histopathological diagnosis, expression of p63 and AMACR immunostaining gives highly significant $\mathrm{p}$ value of $0.001(<0.05)$.
\end{abstract}

Conclusion: p63 and AMACR are reliable markers which can be used in morphologically difficult cases.

Keywords: Adenocarcinoma, AMACR, Benign,Prostate, $p 63$

\section{Introduction}

Prostate cancer is the sixth most common cancer in the world, accounts for $9.7 \%$ of cancers in men showing increasing trend worldwide. ${ }^{[1]}$ Significant advances have occurred in the understanding of pre-malignant epithelial lesions as well as new clinical techniques, enhancing early detection of cancer, such as by transrectal ultrasound and serum levels of prostate specific antigen. ${ }^{[2]}$ About $20-40 \%$ of patients have high stage tumours at the time of diagnosis comprising mainly of conventional adenocarcinoma $(90 \%){ }^{[3]}$

The diagnosis of prostatic adenocarcinoma is usually made on morphological grounds; however, certain problem areas exist. ${ }^{[4]}$ First in the separation of well differentiated adenocarcinoma from vast number of benign or atypical small gland proliferations. These lesions include atrophy, atypical adenomatous hyperplasia (adenosis), basal cell hyperplasia, atypical small acinar proliferation and high grade prostatic intraepithelial neoplasia. ${ }^{[5]}$ Second is the threshold for recognising extremely small foci of cancer in needle biopsies.

Cases with ambiguous lesions of prostate or microscopic foci may require immunohistochemical studies. Prostatic adenocarcinoma is typically immunoreactive for prostate specific antigen (PSA), prostate specific acid phosphatase (PSAP) and $\alpha$-methylacyl CoA racemase (AMACR) but negative for $p 63 .{ }^{[6]}$

Nuclear protein p63 is encoded by gene present on chromosome $3 \mathrm{q} 27-29$ which is homologous to p53 (a tumour suppressor gene), helps to regulate growth and development in epithelium of the skin, cervix, breast and urogenital tract. It stains a subset of 34-betaE12 negative basal cells, is less susceptible to the staining variability of 34-betaE 12 (particularly in transurethral resection of prostate (TURP) specimens with cautery artefact), and interpretation is easier because of its strong nuclear staining intensity and low background. ${ }^{[1]}$

$\alpha$-methylacyl CoA racemase (AMACR) is a wellcharacterized enzyme that plays a key role in peroxisomal $\beta$ oxidation of dietary branched chain fatty acids and C27bile acid intermediates which is consistently up regulated in prostatic carcinoma. ${ }^{[7]}$

Since treatment options and prognosis of prostatic adenocarcinomas and benign lesions differ significantly, so, the current study was carried out with aim to evaluate the 
utility of immunohistochemistry in resolving ambiguous lesions of prostate.

\section{Material and Methods}

The present descriptive cross-sectional study was conducted on 130 prostatic specimens from July 2016 to September 2018 at a tertiary care teaching hospital in western India. Prostatic specimens included prostate biopsy, TURP and prostatectomy specimens.

The histology slides were prepared from paraffin blocks and were subjected to routine Ehrlich's hematoxylin and eosin stain. For Immunohistochemical staining AMACR and p63 monoclonal antibody markers provided by BioGenex, CA (USA), was performed according to the IHC protocol. The sections were examined, brown nuclear staining of basal cells was considered positive for p63 and dark, diffuse cytoplasmic staining of glandular epithelium for AMACR.

These cases were thoroughly evaluated with reference of age, duration of signs and symptoms of urinary hesitancy, frequency, digital rectal examination findings, type of surgery. Lab investigations with serum PSA levels, transrectal ultrasonography and other special investigations were correlated with histopathological findings whenever available.

\section{Results}

Total Prostatic samples accounts $1.7 \%$ of all surgical specimens received in our institute. Out of which 130 cases of prostate were studied, comprising 102 (78.5\%) benign cases and $28(21.5 \%)$ malignant cases. These include TURP (58.5\%), biopsy (36.2\%) and simple prostatectomy $(5.3 \%)$.

In present study, most of the incidence of both benign and malignant carcinoma was seen in age group of 6170 years followed by 71-80 years of age group (25.4\%). Most common symptom in benign lesions and malignant carcinoma was increased urinary frequency and difficulty in micturition respectively. Hard prostate was significantly associated with malignant cases on Digital rectal examination (DRE). PSA level ranging between 0-4 $\mathrm{ng} / \mathrm{ml}$ was seen in 64 cases out of 102 benign prostatic lesions and serum PSA $>20 \mathrm{ng} / \mathrm{ml}$ was seen in 21 cases of malignant carcinoma. The lowest serum PSA value noted in carcinoma was $1.14 \mathrm{ng} / \mathrm{ml}$ seen in poorly differentiated carcinoma with Gleason score of 5+4=9 (Grade V).

$\mathrm{BPH}$ was the most frequent finding and was observed in 102 cases $(78.5 \%)$, composed of varying proportions of glandular epithelium and stroma. Other commonly encountered findings in BPH were metaplasia's, inflammation and atrophy. Nodular hyperplasia with prostatitis was seen in 56 cases $(54.9 \%)$ showing diffuse infiltration of glands and stroma by mononuclear infiltrate predominantly lymphocytes and few plasma cells and histiocytes. Nodular hyperplasia alone was seen in 32 cases. Acute prostatitis with or without necrosis was seen in 2 cases $(2 \%)$. Acute and chronic inflammation with reactive nuclear atypia and occasional prominent nucleoli, may result in both architectural and cytological abnormalities that may be confused with carcinoma, noted in present study. No case of tuberculous inflammation was found. Basal cell hyperplasia was seen in 7 cases $(6.9 \%)$ [Figure 1], squamous metaplasia in only 4 cases $(3.9 \%)$ and 1 case $(0.9 \%)$ of mucinous metaplasia with BPH was noted. Corpora amylacea was present in most of the cases of BPH. [Figure-2].

Prostatic intraepithelial neoplasia was seen in 17 cases comprising 15 cases (11.5\%) of LGPIN and 2 cases $(1.5 \%)$ of HGPIN. All cases of LGPIN were histologically associated with BPH and HGPIN were associated with prostatic adenocarcinoma. Out of 28 cases of prostatic adenocarcinoma, mainly Gleason score $\leq 6$ (Grade I) was seen in 8 cases $(28.6 \%)$ followed by Gleason score 9-10 (Grade V) in 7 cases (25\%) and Gleason score $4+3=7$ (Grade III) in 6 cases $(21.4 \%)$. Perineural invasion was noted in $1(3.6 \%)$ case.

All benign cases comprising mainly BPH showed p63 complete positive [Figure 3] and AMACR was complete negative in 97 cases. However, in 5 cases, there was focal and weak luminal staining seen in benign glands which were interpreted as negative. LGPIN with BPH was seen in 15 cases amongst 130 cases studied showed complete positivity in 12 cases $(80 \%)$ and partial positivity in 3 cases (20\%) with p63 immunostaining [Figure 4]. HGPIN with prostatic adenocarcinoma were seen in 2 cases out of 130 cases studied, which shows focal positivity with expression of p63 immunostaining. Out of all the 28 malignant cases AMACR was positive in 27 cases and negative in 1 case [Figure 5,6], whereas all cases of adenocarcinoma showed complete absence of p63 expression (100\%) [Figure 7].

Comparative study was done between DRE, PSA, final histopathological diagnosis, expression of p63 and AMACR immunostaining giving highly significant $p$ value of 0.001 $(<0.05)$. Positive AMACR and negative p63 staining were noted in 3 cases of malignant carcinoma which were firm on DRE whereas 5 cases of benign lesions which were hard on DRE showed presence of p63 marker. Cases with moderate elevation of PSA seen in reactive prostate and BPH showed complete or partial positivity with p63 expression whereas poorly differentiated carcinoma with 
serum PSA $1.14 \mathrm{ng} / \mathrm{ml}$ showed negative $\mathrm{p} 63$ and positive AMACR expression.

Sensitivity and specificity of p63 and AMACR were calculated comparing results of the $\mathrm{H}$ and $\mathrm{E}$ (gold standard) with benign and malignant lesions. In our study we have concluded that p63 has a sensitivity of $92.86 \%$ and specificity of $100 \%$ [Table 1] whereas AMACR has a sensitivity of $96.4 \%$ and specificity of $95 \%$ [Table 2].

\section{Discussion}

Prostate cancer is the leading cause of new cancer in men after lung cancer as cause of cancer related deaths in men. ${ }^{\left[{ }^{[3}\right.}$ Advancing age and continues androgen supply are the risk factors for BPH. The development of symptoms in BPH depends on glandular epithelium to stroma proliferation. ${ }^{[9]} \mathrm{PIN}$ and AAH are now considered to be most common precancerous lesions of prostate cancer. ${ }^{[10]}$ In view of increasing occurrence of both malignant and benign lesions of prostate, a prospective study was undertaken.

The age of the patients in our study ranged from 35 years to 90 years; however predominant population was in age group of $61-70$ years (45.4\%). Total of 130 cases was studied, out of which 102 were benign (78.5\%) and 28 were malignant $(21.5 \%)$, showing that benign tumors form the bulk of cases. These findings were in concordance with the studies done by Thaker BD et al ${ }^{[11]}$, Sharma A et al ${ }^{[10]}$, Puttaswamy K et al ${ }^{[12[}$, Kumar M et $\mathrm{al}^{[13]}$, Khatib W et $\mathrm{al}^{[14]}$, Chandanwale $\mathrm{S}$ et $\mathrm{al}^{[15]}$ and $\mathrm{Garg} \mathrm{M}$ et $\mathrm{a}^{\left[{ }^{[16]}\right.}$.

Most common clinical presentation encountered in these patients were increased frequency of micturition in benign lesions and difficulty in micturition in malignant

Table 1: Sensitivity and Specificity of p63.

\begin{tabular}{|l|l|l|l|l|}
\hline \multirow{2}{*}{ p63 } & \multicolumn{4}{|c|}{ H \& E (Gold Standard) } \\
\cline { 2 - 5 } & Result & Malignant & Benign & Total \\
\cline { 2 - 5 } & Malignant & $26(\mathrm{TP})$ & $0(\mathrm{FP})$ & 26 \\
\cline { 2 - 5 } & Benign & $2(\mathrm{FN})$ & $102(\mathrm{TN})$ & 104 \\
\cline { 2 - 5 } & Total & $\mathbf{2 8}$ & 102 & 130 \\
\hline
\end{tabular}

Sensitivity $=(T P / T P+F N) \times 100=(26 / 26+2) \times 100=92.86 \%$, Specificity $=(T N / T N+F P) X 100=(102 / 102+0) \times 100=100 \%$

Table 2: Sensitivity and Specificity of AMACR

\begin{tabular}{|c|c|c|c|c|}
\hline & $H \& E(G c$ & & & \\
\hline \multirow[t]{4}{*}{ AMACR } & Result & Malignant & Benign & Total \\
\hline & Malignant & $27(\mathrm{TP})$ & $5(\mathrm{FP})$ & 32 \\
\hline & Benign & $1(\mathrm{FN})$ & 97 (TN) & 98 \\
\hline & Total & 28 & 102 & 130 \\
\hline
\end{tabular}

Sensitivity $=(T P / T P+F N) X 100=(27 / 27+1) \times 100=96.4 \%$, Specificity $=(T N / T N+F P) X 100=(97 / 97+5) \times 100=95 \%$

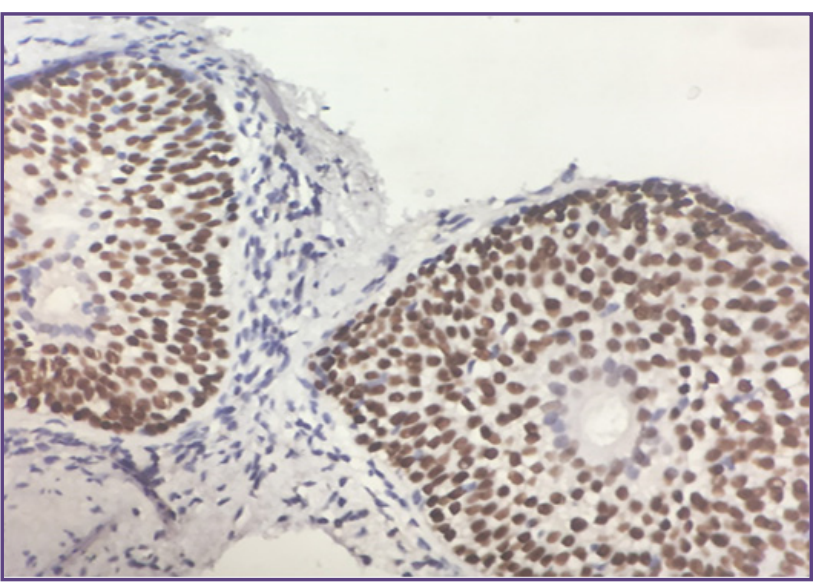

Fig. 1: The proliferating basal cells are immunoreactive for p63- BCH (400X).

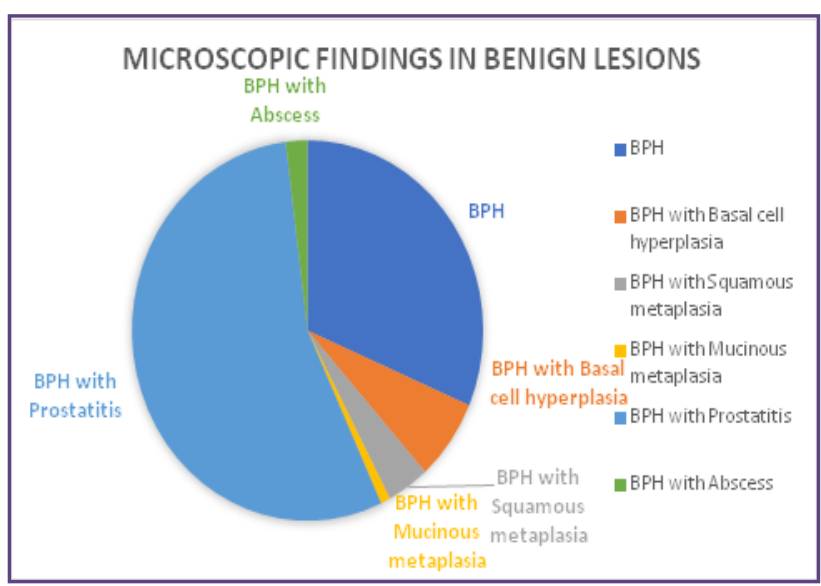

Fig. 2: Microscopic findings in benign lesions in present study. 


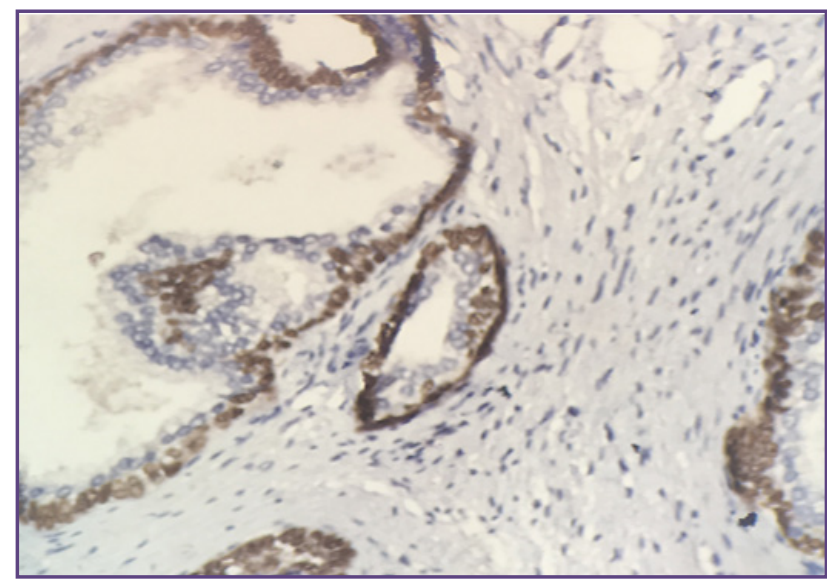

Fig. 3: IHC photomicrograph of p63 in BPH case showing continuous, nuclear $\mathrm{p} 63$ positivity $(400 \mathrm{X})$.

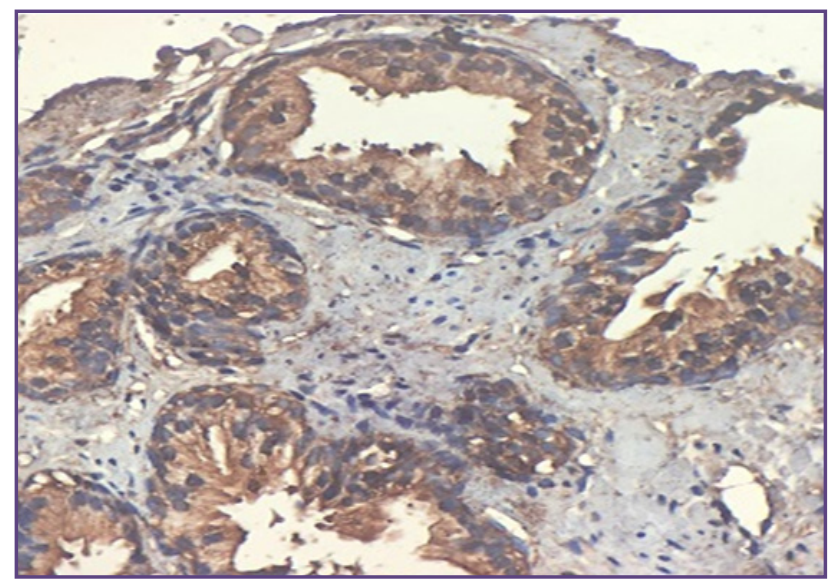

Fig. 5: IHC photomicrograph of AMACR in Prostatic Adenocarcinoma case showing diffuse, cytoplasmic AMACR positivity(400X).

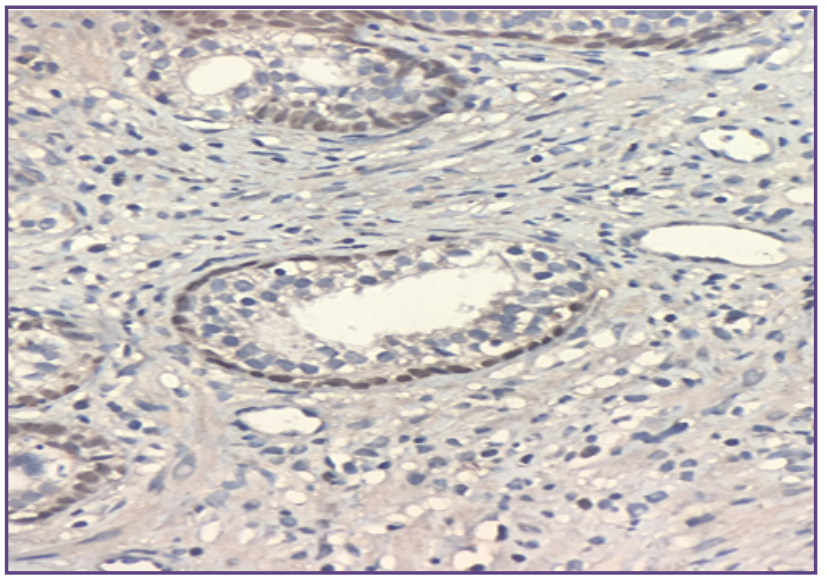

Fig. 4: Photomicrograph showing partial nuclear positivity of p63-LGPIN (400X).

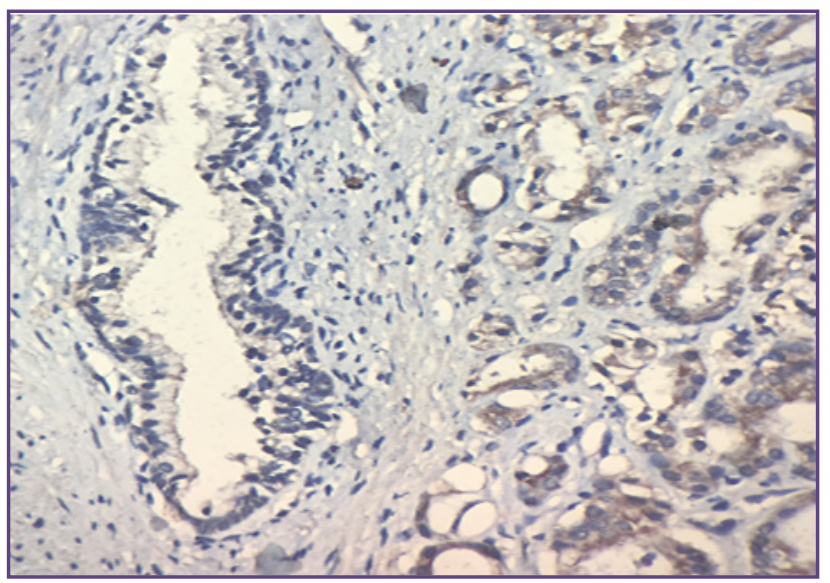

Fig. 6: IHC photomicrograph of AMACR in a case of Prostatic Adenocarcinoma with HGPIN showingcytoplasmic positivity of AMACR in carcinoma and absence of cytoplasmic positivity in HGPIN (400X).

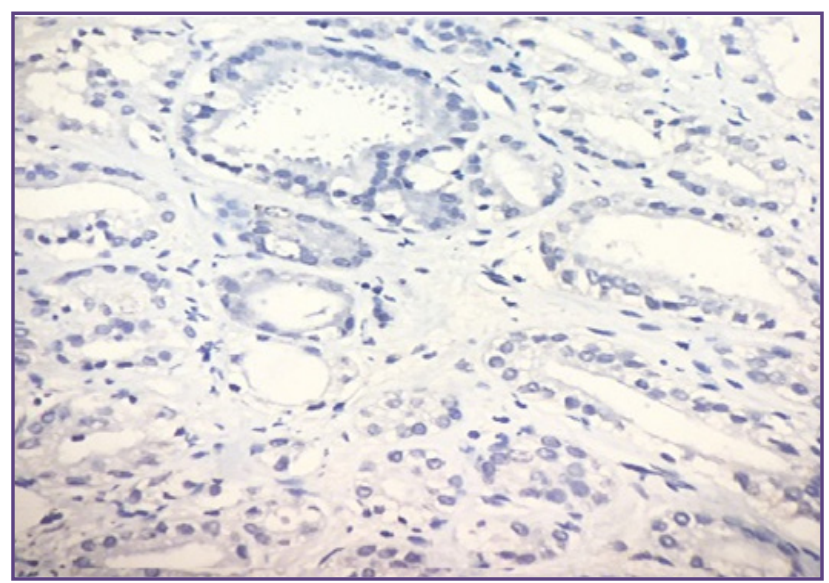

Figure 7: IHC photomicrograph of p63 in prostatic adenocarcinoma case showing absence of p63 nuclear positivity (400X) 
carcinoma similar to studies done by Thaker BD et $\mathrm{a}^{[11]}$ and Anushree $\mathrm{CN}$ et al ${ }^{[17]}$. However, Puttaswamy K et al ${ }^{[12]}$ found hesitancy to be most common symptom followed by frequency in his study. The studies conducted by Kumar $\mathrm{M}$ et $\mathrm{al}^{\left[{ }^{[13]}\right.}$ and Chandanwale $\mathrm{S}$ et $\mathrm{al}^{[15]}$ showed maximum number of patients presented with obstructive urinary tract symptoms which were in contrast with present study.

Digital rectal examination (DRE) is one of the routine screening used in elderly male population along with transrectal ultrasound and serum PSA estimation. American Cancer Society recommends that men $\geq 50$ years should get these tests yearly done. In men with at least one first degree relative with prostate cancer, screening should be started from the age of 45 years. ${ }^{[18]}$ After comparing both benign and malignant lesion, present study concluded hard prostate was significantly associated with malignant cases.

Prostate specific antigen (PSA) is the best marker for adenocarcinoma with high sensitivity and low specificity in patients presenting with obstructive symptoms, having nodules on digital rectal examination. ${ }^{[15]}$ Out of 102 cases of benign prostatic lesions, majority of the 64 cases seen in $0-4 \mathrm{ng} / \mathrm{ml}$ in benign lesions. In malignant lesions, majority ( 21 cases) showed serum PSA $>20 \mathrm{ng} / \mathrm{ml}$. Findings were similar to studies conducted by Kumar M et a ${ }^{13}$, Khatib $\mathrm{W}$ et $\mathrm{al}^{[14]}$, Chandanwale $\mathrm{S}$ et $\mathrm{a}^{\left[{ }^{[15]}\right.}$, Garg $\mathrm{M}$ et $\mathrm{al}^{[16]}$ and Anushree $\mathrm{CN}$ et al ${ }^{[17]}$. The lowest serum PSA value noted in carcinoma was $1.14 \mathrm{ng} / \mathrm{ml}$ seen in poorly differentiated carcinoma with Gleason score of $5+4=9$ (Grade V) and moderate elevation of PSA was also noted in reactive prostate and $\mathrm{BPH}$.

Transurethral resection of prostate (TURP) was the most common specimen encountered in studies done by Puttaswamy K et al ${ }^{[12]}$, Khatib W et a ${ }^{[14]}$ and Chandanwale $\mathrm{S}$ et a ${ }^{[15]}$ similar to present study.

Prostatitis was a common microscopic finding in majority of cases with BPH in concordance to studies done by Thaker BD et al ${ }^{[11]}$, Sharma A et al ${ }^{[10]}$, Khatib W et al ${ }^{[14]}$ and Garg M et al ${ }^{[16]}$. Higher incidence of prostatitis was noted. These differences could be because of inter-observer variation or various management strategies followed at different institutes. Antibiotics and anti-inflammatory may modify the inflammatory pathology on a biopsy.

Acute and chronic inflammation with reactive nuclear atypia and occasional prominent nucleoli, may result in both architectural and cytological abnormalities that may be confused with carcinoma, noted in present study. The distinction of these inflammatory atypia's from carcinoma relies on the recognition that the atypical glands are in an area of intense inflammation. In addition, gland have very basophilic appearance in contrast to usual gland forming prostatic adenocarcinomas that have abundant often pale cytoplasm. The high nuclear to cytoplasmic ratio seen in inflamed glands are predominantly seen in poorly differentiated prostatic carcinomas that lack gland formation. Careful examination of these basophilic glands will demonstrate the presence of basal cell layer in most cases, which can be confirmed by p63 immunostaining.

PIN shows neoplastic transformation of the lining epithelium of prostatic ducts and acini, which is confined within the epithelium. 2 cases of HGPIN were histologically associated with prostatic adenocarcinoma. Holds true for the study conducted by Sharma A et al ${ }^{[10]}$, Puttaswamy K et al ${ }^{[12]}$, Kumar $\mathrm{M}$ et al ${ }^{[13]}$, Chandanwale $\mathrm{S}$ et al ${ }^{[15]}$ and Garg $\mathrm{M}$ et $\mathrm{al}^{[16]}$. It is suggested that patients with HGPIN need close follow up observations and investigations to rule out existence of carcinoma, especially in the peripheral zone. [10]

The Gleason's microscopy grading of prostatic adenocarcinoma was adopted out of which the most common score was $\leq 6$ (Grade I) compare to studies done

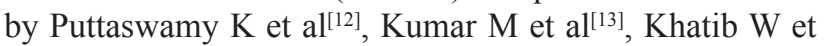
$\mathrm{a}^{\left[{ }^{[14]}\right.}$ and Chandanwale $\mathrm{S}$ et $\mathrm{a}^{\left[{ }^{15]}\right.}$. This could be because of early access to the health care facility as people from the urban area are literate and have better socioeconomic status.

IHC is useful to differentiate low grade prostatic cancer from benign mimics and in metastatic tumor samples to establish the origin of a poorly differentiated carcinoma. In recent years, IHC using monoclonal antibodies against basal cell markers like p63, HMWCK (34ßE12) and prostatic adenocarcinoma specific biomarker AMACR have been used as adjuvant to morphology in diagnostically challenging cases with a very high sensitivity and specificity. This has increased the diagnostic accuracy of prostate cancer world-wide. Basal cell markers are very useful in demonstration of basal cells as their presence hints against a diagnosis of invasive prostatic adenocarcinoma. ${ }^{[7]}$

All benign cases comprising mainly BPH showed p63 complete positive and AMACR was complete negative in 97 cases. However, in 5 cases, there was focal and weak luminal staining seen in benign glands which were interpreted as negative. LGPIN with BPH was seen in 15 cases amongst 130 cases studied showed complete positivity in 12 cases $(80 \%)$ and partial positivity in 3 cases $(20 \%)$ with p63 immunostaining. HGPIN with prostatic adenocarcinoma were seen in 2 cases out of 130 cases studied, which shows focal positivity with expression of p63 immunostaining. Out of all the 28 malignant cases AMACR was positive in 27 cases and negative in 1 case. 
These findings were similar to studies done by Singh V et $\mathrm{al}^{7}$ and Bai EL et al. ${ }^{[19]}$

\section{Conclusion}

Combination of p63 and AMACR plays an important role in the diagnosis of ambiguous prostatic lesions, further improving the treatment outcome of patients. Comparative study done between DRE, PSA, final histopathological diagnosis, expression of p63 and AMACR immunostaining gives highly significant $\mathrm{p}$ value of $0.001(<0.05)$.

\section{Acknowledgements}

I would like to express my sincere gratitude to my guide, parents, brother and my college for their belief, timely encouragement, unconditional love and support.

\section{Funding}

Self-funded

\section{Competing Interest}

No conflict of interest

\section{References}

1. Eble JN, Sauter G, Epstein JI, Sesterhenn IA. Pathology and genetics of tumours of the urinary system and male genital organs. Lyon: IARC Press; 2004. p.162-92.

2. Obiorah CC, Nwosu SO. A histopathological study of carcinoma of the prostate in Port Harcourt, Nigeria. Niger J Clin Pract 2011; 14(3):363-7.

3. Templeton H. Prostate cancer- presentation, diagnosis and treatment: what does the literature say? Int J Urol Nurs. 2007; 1(1):6-17.

4. Humphery PA. Diagnosis of adenocarcinoma in prostate needle biopsy tissue. J Clin Pathol. 2007; 60:35-42.

5. Sringley JR. Benign mimickers of prostatic adenocarcinoma. Mod Pathol. 2004; 17:328-48.

6. Gunia S, Koch S, May M, Dietel M, Erbersdobler A. Expression of prostatic acid phosphatase (PSAP) in transurethral resection specimens of the prostate is predictive of histopathologic tumour stage in subsequent radical prostatectomies. Virchows Arch 2009; 454(5):573-9.
7. Singh V, Manu V, Malik A, Dutta V, Mani NS, Patrikar S. Diagnostic utility of p63 and $\alpha$-methyl acyl CoA racemase in resolving suspicious foci in prostatic needle biopsy and transurethral resection of prostate specimens. J Cancer Res Ther. 2014; 10(3):686-92.

8. Hsing AW, Chokkalingram AP. Prostate cancer epidemiology. Front Biosci 2006; 11(2):1388-413.

9. Shapiro E, Becich MJ, Hartanto V. The relative proportion of stromal and epithelial hyperplasia is related to development of symptomatic benign prostate hyperplasia. J Urol.1992; 147:1293-1296.

10. Sharma A, Sharma M, Gandhi S, Khajuria A, Goswami KS Int J Res Med Sci. 2017;5(6):2373-2378.

11. Thaker BD, Raina S, Singh $K$. Histopathological spectrum of prostatic lesion: a hospital based study.GJRA. 2017;6(7).

12. Puttaswamy K, Prathibhan R, Shariff S. Histopathological study of prostatic biopsies in men with prostatism. J Med. Sci. Health,2016;2(1):11-17.

13. Kumar M, Khatri SL, Saxena V,Vijay S. Clinicopathological study of prostatic lesions. IJBAMR. 2016; 6:695-704.

14. Khatib W, Jagtap S, Demde R, Shukla DB, Bisht T. Clinicopathological study of prostate lesions-A one year study. Int J Med Res Health Sci. 2016,5(5):183-186.

15. Chandanwale S, Jadhav SP, Anwekar CS, Kumar H, Buch CA, Chaudhari SU. Clinicopathological Study of Benign \& Malignant Lesions of Prostate. IJPBS. 2013;3(1):162-178.

16. Garg M, Kaur G, Malhotra V, Garg R. Histomorphological spectrum of 364 prostatic specimens including immunohistochemistry with special reference to grey zone lesions. Prostate Int. 2013;1(4):146-151.

17. Anushree C.N., Venkatesh Kusuma. Morphological Spectrum of Prostatic Lesions- A Clinicopathological Study. Medica Innovatica 2012; 1(2): 49-54.

18. Schmitz M. American cancer society prostate cancer screening guidelines. About, com, Updated January 21,2010.

19. Bai EL, Sandhya S, Annapurna P, Rani HS. A Histomorphological Study of Prostatic TURP Specimens with Special Reference to Prostatic Intraepithelial Neoplasia by Using Immunohistochemistry. IJAR. 2018;6. 